\title{
Prospective Evaluation of Health-Related Quality of Life in Patients With Deep Venous Thrombosis
}

\author{
Susan R. Kahn, MSc, MD, FRCPC; Thierry Ducruet, MSc; Donna L. Lamping, PhD; Louise Arsenault; \\ Marie Jose Miron, MD; Andre Roussin, MD; Sylvie Desmarais, MD; France Joyal, MD; Jeannine Kassis, MD; \\ Susan Solymoss, MD; Louis Desjardins, MD; Mira Johri, PhD; Ian Shrier, MD, PhD
}

\begin{abstract}
Background: To our knowledge, the burden of deep venous thrombosis from the patient's perspective has not been quantified. We evaluated health-related quality of life (QOL) after deep vein thrombosis and compared results with general population norms.
\end{abstract}

Methods: This was a multicenter prospective cohort study of 359 consecutive eligible patients with deep vein thrombosis recruited at 7 Canadian hospital centers. Quality of life was assessed at baseline and at 1 and 4 months after diagnosis using generic (36-Item Short-Form Health Survey) and disease-specific (Venous Insufficiency Epidemiological and Economic Study [VEINES]-QOL and VEINES symptom [VEINES-Sym] questionnaires) measures. Changes in QOL scores during the 4-month period were calculated, and determinants of lack of improvement in QOL were evaluated.

Results: During the 4 months, mean 36-Item ShortForm Health Survey physical and mental component summary scores improved by 5.1 and 4.6 points, respec- tively, and VEINES-QOL and VEINES-Sym scores improved by 3.1 and 2.2 points, respectively $(P<.001$ for time trend for all measures). However, about one third of patients had worsening of QOL during follow-up. Multivariate analyses showed that worsening of the postthrombotic syndrome score was an independent predictor of worsening of 36-Item Short-Form Health Survey physical component summary $(P=.04)$, VEINES-QOL $(P<.001)$, and VEINES-Sym $(P<.001)$ scores. The 36Item Short-Form Health Survey physical component summary scores were lower than population norms at all points assessed.

Conclusions: On average, QOL improves during the 4 months following deep vein thrombosis. However, in about one third of patients, QOL deteriorates, and at 4 months, average QOL remains poorer than population norms. Worsening of the postthrombotic syndrome score is associated with worsening of QOL.

Arch Intern Med. 2005;165:1173-1178
Author Affiliations: Centre for Clinical Epidemiology and Community Studies, Sir Mortimer B. Davis Jewish General Hospital (Drs Kahn, Johri, and Shrier, Mr Ducruet, and Ms Arsenault), Departments of Medicine (Drs Kahn and Solymoss) and Family Medicine (Dr Shrier), McGill University, and Départements de Médecine (Drs Miron, Roussin, Joyal, and Kassis) et d'administration de la santé (Dr Johri), Université de Montréal, Montreal, Quebec; Health Services Research Unit, London School of Hygiene \& Tropical Medicine, London, England (Dr Lamping); Centre Hospitalier Pierre Boucher, Longueuil, Quebec (Dr Desmarais); and Université Laval, Québec (Dr Desjardins). Financial Disclosure: None.

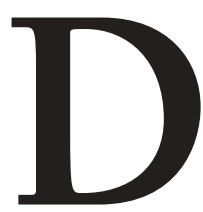

EEP VENOUS THROMBOSIS (DVT) is a common cardiovascular condition with substantial morbidity and mortality. Studies of the natural history or treatment of DVT have traditionally evaluated clinical end points such as recurrence of venous thromboembolism. While important, such end points are limited in their ability to describe and quantify the full impact of illness on patients' health and daily functioning. Quality of life (QOL), by defining health in broader terms than morbidity and mortality, yields valuable information on burden of illness and is an important end point to consider when studying patients with DVT. ${ }^{1-4}$ To date, however, the impact of DVT on QOL has received little attention by thrombosis investigators.

During a Canadian multicenter prospective study of long-term outcomes after DVT, we evaluated temporal patterns and deter- minants of change in generic and venous disease-specific QOL during the 4 months following DVT. In addition, we compared QOL scores in our patients with norms previously reported for the general Canadian population and for patients with other types of medical conditions.

\section{METHODS}

\section{STUDY POPULATION}

The study population was recruited from among consecutive eligible patients with acute symptomatic DVT seen in the emergency departments, outpatient clinics, and inpatient wards of 7 participating hospital centers in Quebec. Patients were potentially eligible to participate in the study if they had objectively confirmed DVT of the lower limb and if they were able to read and understand English or French. The diagnosis of DVT was based on a venous duplex study showing lack of compressibility or intralumi- 
nal thrombus in the calf or proximal veins ${ }^{5}$ or a contrast venogram showing a constant intraluminal filling defect in at least 2 different projections. ${ }^{6}$ Eligible patients were excluded if their expected lifespan was less than 3 months, they were incapable of responding to a questionnaire, they were geographically inaccessible or unwilling to return for follow-up visits, or they were unable or refused to provide informed consent. Excluded patients were asked permission to record basic demographic and clinical data to assess their comparability with included patients.

Ethics approval was obtained from the relevant committees in each hospital center, and written informed consent was obtained from all patients before study enrollment.

\section{STUDY PROCEDURES}

Standard treatment of DVT at all centers during the period of study recruitment and follow-up included subcutaneous lowmolecular-weight heparin (or intravenous unfractionated heparin, in hospitalized patients) for 5 to 10 days, followed by oral warfarin sodium for 3 months or longer, per consensus practice guidelines. ${ }^{7}$ Management of acute DVT was left to the judgment of the treating physicians at each study center.

Study patients were examined at clinic visits that took place at enrollment (baseline) and at 1 and 4 months after diagnosis. At clinic visits, clinical and demographic data were collected, the study nurse or physician performed a clinical examination to evaluate symptoms and signs of the postthrombotic syndrome, and patients self-completed generic and venous disease-specific QOL questionnaires, as described later.

\section{Clinical and Demographic Data}

Age, sex, body mass index (BMI), patient location (inpatient vs outpatient), level of education, employment, comorbid conditions, DVT risk factors, prior venous thromboembolism, medications, and details regarding diagnosis of the index DVT were recorded.

\section{Assessment of the Postthrombotic Syndrome Score}

Symptoms and signs of the postthrombotic syndrome were assessed in the ipsilateral leg by the research nurse or physician using a validated clinical scale developed by Villalta et al. ${ }^{8}$ This scale grades the severity, from 0 (absent) to 3 (severe), of each of 5 symptoms (pain, cramps, heaviness, pruritus, and paresthesia) and 6 signs (edema, skin induration, hyperpigmentation, venous ectasia, redness, and pain during calf compression). The maximum summative score is 33. A score of 5 or more or the presence of a venous ulcer is indicative of the postthrombotic syndrome.

\section{Assessment of QOL}

Standardized self-administered instruments were used to measure generic and venous disease-specific QOL. These included the 36-Item Short-Form Health Survey $(\mathrm{SF}-36)^{9}$ and the Venous Insufficiency Epidemiological and Economic Study (VEINES)-QOL, ${ }^{10}$ respectively. The SF-36 is the generic gold standard measure of QOL. Two summary scores are produced (physical component summary [PCS] and mental component summary [MCS] scores), which reflect physical and mental health status, respectively. The VEINES-QOL is a 26-item questionnaire that measures venous symptoms (heavy legs, aching legs, swelling, night cramps, heat or burning sensation, restless legs, throbbing, itching, tingling, and intensity of leg pain), limitations in daily activities due to chronic venous disease, psychological impact of chronic venous disease, change during the past year, and time of day leg problem is most intense. The VEINES-Sym is a validated subscale of the VEINES-QOL that measures venous symptoms. English- and French Canadianlanguage versions of the VEINES-QOL have been comprehensively evaluated and shown to be acceptable, reliable, valid, and responsive. ${ }^{10}$ For all measures, lower scores indicate poorer QOL.

\section{STATISTICAL ANALYSIS}

Mean scores for the SF-36 PCS, SF-36 MCS, VEINES-QOL, and VEINES-Sym were computed in the study population at baseline and at the 1- and 4-month follow-ups using standard scoring algorithms. ${ }^{10,11}$ Differences in mean scores over time were analyzed using repeated-measures analysis of variance, where positive change scores indicated improvement in QOL. $P<.05$ was considered to represent a statistically significant difference. Proportions of patients who worsened from the previous time point (defined as negative mean change in QOL score) were calculated. Potential determinants of worsening in QOL scores from 1 to 4 months were evaluated using multivariable logistic regression analyses. We chose to evaluate change during this period rather than the baseline to 4-month period because the time frame for questions in the QOL measures is the preceding 4 weeks. Hence, scores at baseline (ie, time of study enrollment) are likely to reflect a composite of pre-DVT- and acute DVT-related QOL.

Expected general population scores for the SF-36 PCS and MCS were calculated based on norms reported for the general Canadian adult population, ${ }^{12}$ standardized to the study cohort's distribution of sex and age deciles. The SF-36 PCS and MCS scores obtained in study patients were compared with expected general population scores, and with scores reported for US patients with arthritis, chronic lung disease, and angina. ${ }^{9}$

\section{RESULTS}

\section{PATIENT CHARACTERISTICS}

From April 23, 2001, to July 5, 2002, 1004 consecutive patients objectively diagnosed as having DVT were approached for participation in the study. Of these patients, 645 were excluded for the following reasons: refusal or inability to provide informed consent $(n=308)$, incapable of responding to a questionnaire in English or French $(n=138)$, estimated life expectancy of less than 3 months $(n=116)$, geographic inaccessibility for follow-up $(n=80)$, and other $(n=3)$. Compared with enrolled patients, those excluded were older (mean age, 65 vs 56 years; $P<.001$ ), were more likely to be women ( $59.5 \%$ vs $50.1 \% ; P=.003)$, and were more likely to have proximal DVT $(69.6 \%$ vs $54.9 \%$; $P<.001)$.

Baseline characteristics of the 359 patients enrolled in the study cohort are shown in Table 1. Half of the patients were men, the mean age was 56 years, and two thirds were outpatients. Deep vein thrombosis was located in proximal venous segments in $54.9 \%$ of the patients, and in distal (infrapopliteal) segments in 45.1\%. Concurrent symptomatic pulmonary embolism (PE) was present in $15.2 \%$ of patients, and previous venous thromboembolism was documented in $21.7 \%$ of patients.

\section{CLINICAL FOLLOW-UP}

At the 1- and 4-month follow-up visits, 93.8\% and 36.7\% of patients, respectively, were still taking warfarin. Elas- 
tic stocking use of any frequency was reported by $65.8 \%$ of patients at the 1 -month visit and $42.4 \%$ of patients at the 4-month visit. During the 4-month follow-up, 10 patients died and there were 9 objectively confirmed recurrent venous thromboembolism events (DVT, 3; PE, 5; and both DVT and PE, 1).

The mean (SD) postthrombotic syndrome score was 5.0 (3.9) at 1 month and 4.2 (4.0) at 4 months, and the percentage of patients with a postthrombotic syndrome score of 5 or more was $46.8 \%$ at 1 month and $38.7 \%$ at 4 months. Whether the DVT was proximal or distal did not influence these results (data not shown). Worsening of the postthrombotic syndrome score from 1 to 4 months was documented in $48.4 \%$ of the study patients.

\section{BASELINE QOL}

Baseline QOL scores for the entire cohort and for patient subgroups are shown in Table 2. For all QOL measures, women had significantly lower scores than men. The SF-36 (generic QOL) scores were significantly lower among inpatients and patients with a comorbidity than among outpatients and patients without a comorbidity. Patients with a higher BMI had significantly lower SF-36 PCS, VEINES-QOL, and VEINES-Sym scores than did patients with a lower BMI. The SF-36 PCS scores were lower in patients with proximal DVT than in patients with distal DVT; however, QOL scores were not influenced by whether DVT was first or recurrent or accompanied by symptomatic PE.

\section{CHANGE IN QOL}

Quality-of-life scores were available at all 3 time points for 310 study patients. There were no significant changes in mean QOL scores from the enrollment (baseline) visit to the 1-month visit. However, from the 1- to the 4-month visit, the mean SF-36 PCS score improved by 5.1 points, the mean SF-36 MCS score improved by 4.6 points, the mean VEINES-QOL score improved by 3.1 points, and the mean VEINES-Sym score improved by 2.2 points $(P<.001$ for time trend for all 4 QOL measure) (Figure 1 ).

The proportions of patients whose QOL worsened during study follow-up are shown in Figure 2. From the 1- to the 4-month visit, worsening of SF-36 PCS, SF-36 MCS, VEINES-QOL, and VEINES-Sym scores was documented in $30.3 \%, 34.2 \%, 22.2 \%$, and $29.3 \%$ of patients, respectively. Among patients whose scores worsened, the mean number of points decreased in score for the 4 measures was 5.9, 5.9, 3.8, and 2.8, respectively, and the proportion of patients whose score worsened by 4 or more points was $53.7 \%, 50.4 \%, 24.6 \%$, and $36.2 \%$ for the 4 measures, respectively. As a point of reference, 4- to 5-point differences in SF-36 scores between groups are considered to indicate clinically relevant change. ${ }^{11}$

\section{PREDICTORS OF WORSENING OF QOL}

Multivariate logistic regression analyses were performed to evaluate potential predictors of worsening of QOL during study follow-up. In separate models, the dependent variable was change in score, dichotomized as improved vs worse, for each of the QOL measures (SF-36

\begin{tabular}{|lc}
\hline \multicolumn{2}{|l}{ Table 1. Baseline Characteristics of the 359 Study } \\
Participants \\
\hline Characteristic & \multicolumn{1}{l}{ Value* } \\
\hline Male sex & $180(50.1)$ \\
Age, y† & $55.8(14.8)$ \\
Patient location & \\
Inpatient & $117(32.6)$ \\
Outpatient & $242(67.4)$ \\
Highest level of education & \\
No schooling & $2(0.6)$ \\
Primary school & $34(9.5)$ \\
High school & $123(34.3)$ \\
College or university & $200(55.7)$ \\
Not employed (includes retired, student, & $173(48.2)$ \\
homemaker, and unemployed) & \\
BMI† & $27.7(6.0)$ \\
VTE risk factors $¥$ & \\
Surgery & $100(27.9)$ \\
Trauma & $62(17.3)$ \\
Immobilization & $79(22.0)$ \\
Active cancer & $45(12.5)$ \\
Estrogenic drugs & $83(23.1)$ \\
Pregnancy & $8(2.2)$ \\
Location of DVT & \\
Proximal & $197(54.9)$ \\
Distal & $162(45.1)$ \\
Concurrent pulmonary embolism & $54(15.0)$ \\
History of VTE & $78(21.7)$ \\
Current smoker & $66(18.4)$ \\
\end{tabular}

Abbreviations: BMI, body mass index (calculated as weight in kilograms divided by the square of height in meters); DVT, deep venous thrombosis; VTE, venous thromboembolism.

*Data are given as number (percentage) of participants unless otherwise indicated. Percentages may not total 100 because of rounding.

†Data are given as mean (SD).

¥Patients could have more than 1 risk factor.

PCS, SF-36 MCS, VEINES-QOL, and VEINES-Sym). For all measures, age, sex, patient location (inpatient vs outpatient), BMI, presence of comorbidity (defined as active cancer or cardiorespiratory conditions), first vs recurrent DVT, site of DVT (proximal vs distal), and concurrent PE were not predictive of worsening of QOL from 1 to 4 months. However, worsening of the postthrombotic syndrome score (defined as positive mean change in the Villalta et $\mathrm{al}^{8}$ score from 1-4 months) was independently associated with worsening of SF-36 PCS, VEINES-QOL, and VEINES-Sym scores $(P=.04, P<.001$, and $P<.001$, respectively), but not with worsening of the SF-36 MCS score $(P=.99)$.

Among patients who had postthrombotic syndrome at 4 months, the proportion whose SF-36 PCS, VEINES-QOL, and VEINES-Sym scores had worsened by 4 or more points during the preceding 3 months was $21.2 \%, 14.3 \%$, and $15.3 \%$, respectively. The corresponding proportions for patients without postthrombotic syndrome were $13.4 \%, 3.7 \%$, and $7.4 \%$, respectively.

\section{QOL IN STUDY PATIENTS COMPARED WITH OTHER POPULATIONS}

Mean SF-36 PCS scores at all 3 visits were substantially lower than Canadian adult norms (Table 3). The mean 
Table 2. Baseline QOL by Patient Characteristics

\begin{tabular}{|c|c|c|c|c|c|c|c|c|c|}
\hline \multirow[b]{3}{*}{ Characteristic } & \multirow{3}{*}{$\begin{array}{c}\text { No. of } \\
\text { Patients }\end{array}$} & \multicolumn{4}{|c|}{ Generic QOL (SF-36 Data) } & \multicolumn{4}{|c|}{ Disease-Specific QOL } \\
\hline & & \multicolumn{2}{|c|}{ PCS } & \multicolumn{2}{|c|}{ MCS } & \multicolumn{2}{|c|}{ VEINES-QOL Scale } & \multicolumn{2}{|c|}{ VEINES-Sym Subscale } \\
\hline & & Score* & $P$ Value & Score* & $P$ Value & Score* & $P$ Value & Score* & $P$ Value \\
\hline Entire cohort & 359 & $38.6(12.7)$ & NA & $46.2(12.1)$ & NA & $50.0(6.2)$ & NA & $50.0(6.8)$ & NA \\
\hline \multicolumn{10}{|l|}{ Sex } \\
\hline Male & 180 & $40.7(12.8)$ & \multirow[t]{2}{*}{.002} & $47.7(12.1)$ & \multirow[t]{2}{*}{.02} & $50.9(6.1)$ & \multirow[t]{2}{*}{.004} & $50.8(6.7)$ & \multirow[t]{2}{*}{.03} \\
\hline Female & 179 & $36.4(12.3)$ & & $44.6(11.8)$ & & $49.0(6.3)$ & & $49.2(6.9)$ & \\
\hline \multicolumn{10}{|l|}{ Patient location } \\
\hline Inpatient & 117 & $35.3(12.5)$ & \multirow[t]{2}{*}{$<.001$} & $43.7(12.2)$ & .008 & $50.2(6.3)$ & \multirow[t]{2}{*}{.68} & $50.8(6.5)$ & \multirow[t]{2}{*}{.10} \\
\hline Outpatient & 242 & $40.3(12.5)$ & & $47.4(11.9)$ & & $49.9(6.2)$ & & $49.6(7.0)$ & \\
\hline \multicolumn{10}{|l|}{ Age, y } \\
\hline$<40$ & 60 & $38.1(14.8)$ & \multirow[t]{3}{*}{.07} & $46.1(10.3)$ & .98 & $49.2(7.2)$ & \multirow[t]{3}{*}{.52} & $49.7(7.5)$ & \multirow[t]{3}{*}{.69} \\
\hline $40-65$ & 184 & $40.1(13.0)$ & & 46.1 (12.1) & & $50.2(6.1)$ & & $50.3(6.7)$ & \\
\hline$>65$ & 115 & $36.6(10.9)$ & & $46.3(13.1)$ & & $49.9(6.1)$ & & $49.7(6.7)$ & \\
\hline \multicolumn{10}{|l|}{ BMI } \\
\hline$<25$ & 113 & $39.5(13.0)$ & \multirow[t]{3}{*}{.01} & $45.1(13.3)$ & .32 & $50.7(6.8)$ & \multirow[t]{3}{*}{.004} & $50.8(7.1)$ & \multirow[t]{3}{*}{.03} \\
\hline $25-30$ & 154 & $40.0(12.0)$ & & $47.3(11.3)$ & & $50.6(6.0)$ & & $50.4(6.7)$ & \\
\hline$>30$ & 92 & $35.2(11.5)$ & & 45.6 (11.9) & & $48.1(5.7)$ & & $48.4(6.4)$ & \\
\hline \multicolumn{10}{|l|}{ Location of DVT } \\
\hline Proximal & 197 & $37.4(12.5)$ & \multirow[t]{2}{*}{.04} & 46.7 (11.9) & 33 & $49.9(6.2)$ & \multirow[t]{2}{*}{.22} & $49.6(6.8)$ & \multirow[t]{2}{*}{.82} \\
\hline Distal & 162 & $40.1(12.9)$ & & 45.4 (12.4) & & $50.1(6.3)$ & & $50.4(6.9)$ & \\
\hline Type of DVT & & & & & & & & & \\
\hline First & 281 & $38.6(13.0)$ & .82 & $45.8(12.2)$ & .27 & $50.0(6.5)$ & .96 & $50.1(7.0)$ & .32 \\
\hline Recurrent & 78 & $38.2(11.7)$ & & $47.5(11.7)$ & & $49.9(5.4)$ & & $49.3(6.4)$ & \\
\hline Concurrent PE & & & & & & & & & \\
\hline Yes & 54 & $37.4(12.3)$ & .46 & $44.2(12.2)$ & .21 & $51.2(6.2)$ & .13 & $50.9(6.5)$ & .29 \\
\hline No & 305 & $38.9(12.8)$ & & $46.5(12.1)$ & & $49.8(6.2)$ & & $49.9(6.9)$ & \\
\hline Comorbidity $\dagger$ & & & & & & & & & \\
\hline Present & 119 & $33.8(10.9)$ & $<.001$ & $43.2(13.0)$ & .001 & $49.3(6.0)$ & .12 & $49.2(6.7)$ & .13 \\
\hline Absent & 240 & $41.0(12.9)$ & & $47.6(11.4)$ & & $50.3(6.3)$ & & $50.4(6.9)$ & \\
\hline
\end{tabular}

Abbreviations: BMI, body mass index (calculated as weight in kilograms divided by the square of height in meters); DVT, deep venous thrombosis; MCS, mental component summary; NA, data not applicable; PCS, physical component summary; PE, pulmonary embolism; Q0L, quality of life; SF-36, 36-Item Short-Form Health Survey; Sym, symptom; VEINES, Venous Insufficiency Epidemiological and Economic Study.

*Data are given as mean (SD).

†Defined as active cancer or cardiac or respiratory disease.

SF-36 PCS score at 1 month was also lower than in patients with arthritis or chronic lung disease, and similar to patients with angina, ${ }^{9}$ and at 4 months was similar to patients with arthritis or chronic lung disease. ${ }^{9}$ For the SF-36 MCS, mean scores at baseline and at 1 month were lower than Canadian adult norms and similar to patients with chronic lung disease, but at 4 months were similar to Canadian adult norms.

\section{COMMENT}

In this multicenter cohort study conducted at 7 hospital centers in Quebec, we prospectively evaluated healthrelated QOL during the 4 months following acute DVT. We found that, on average, generic and venous diseasespecific QOL scores tended to improve over time. However, at 1 and 4 months after diagnosis, SF-36 PCS scores, which reflect physical health status, remained lower than Canadian adult norms and lower or similar to patients with chronic conditions such as arthritis, chronic lung disease, or angina. Furthermore, about one third of patients had worsening of QOL during the 4-month period, indicating that many patients treated for DVT are still burdened by their illness as late as 4 months after diagnosis.
We found that worsening of postthrombotic symptoms and signs, measured using the clinical scale developed by Villalta et al, ${ }^{8}$ was associated with a decline in generic (SF-36 PCS) and venous disease-specific QOL during the 4-month period of follow-up. The postthrombotic syndrome develops in $20 \%$ to $40 \%$ of patients after acute DVT. Despite being the most common complication of DVT, the natural history and timing of onset of the postthrombotic syndrome are not well understood. ${ }^{13}$ At 4 months after DVT, it may be too early to establish whether persistent venous symptoms and signs reflect slow resolution of the acute DVT process or the development of the chronic postthrombotic syndrome. Nevertheless, our findings indicate that a lack of resolution or worsening of venous symptoms and signs during the first 4 months after DVT has a significant adverse impact on generic and disease-specific QOL. We did not find site of DVT (proximal vs distal) to be predictive of change in QOL scores or postthrombotic syndrome scores during the 4-month follow-up. This is in keeping with a recent review ${ }^{13}$ that was unable to demonstrate a consistent association between site of DVT and subsequent development of the postthrombotic syndrome. 


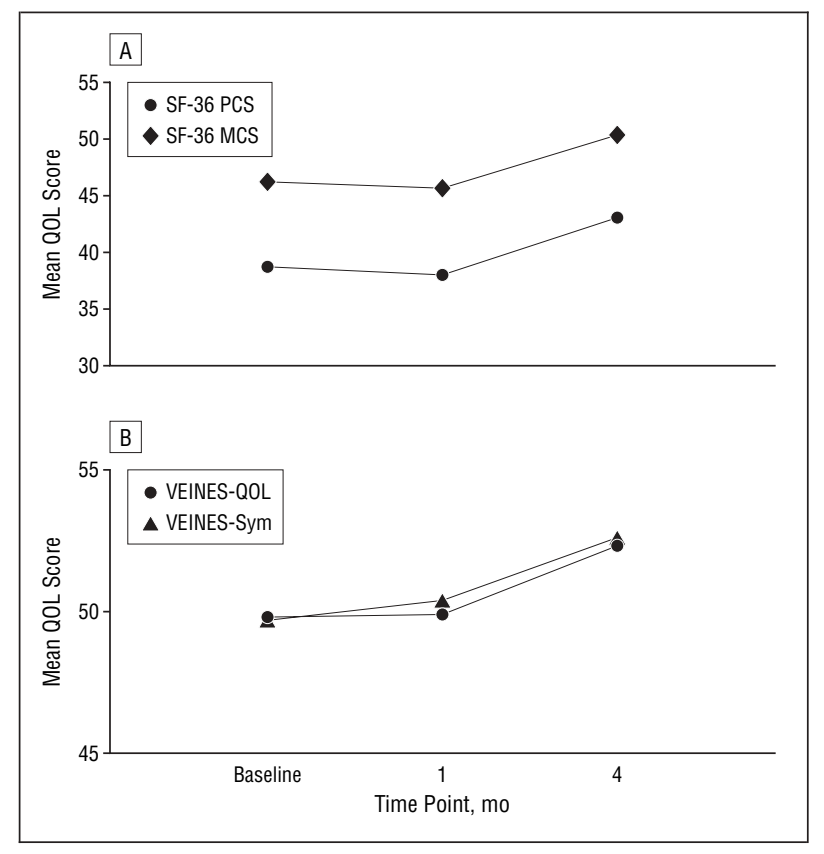

Figure 1. Mean generic (A) and disease-specific (B) quality-of-life (QOL) scores at baseline (enrollment) and at the 1- and 4-month visits. Higher scores indicate a better QOL. $P$ values were not significant for the change in scores from baseline to 1 month for all measures; and $P<.001$ for the change in scores from 1 to 4 months for all measures. SF-36 indicates 36-Item Short-Form Health Survey; PCS, physical component summary; MCS, mental component summary; and VEINES-Sym, Venous Insufficiency Epidemiological and Economic Study Symptom.

Our study has several strengths. We evaluated QOL prospectively starting from the time of DVT diagnosis using measures that have been shown to be reliable, valid, and responsive to change. ${ }^{9,10}$ To ensure comprehensive assessment of QOL, we used generic and diseasespecific measures, because generic measures allow comparisons with general population norms or with groups of patients with other medical conditions, while diseasespecific measures permit assessment of health outcomes within specific diagnostic groups with a focus on measuring changes over time. ${ }^{1,14}$ Consecutive eligible and consenting patients were enrolled, study inclusion criteria were broad, and the management of the acute DVT was consistent across study centers and guided by consensus practice guidelines; hence, our results are likely to be generalizable to other populations of DVT patients. Reasons for nonparticipation and the clinical characteristics of patients who declined participation were documented; hence, the source population for the study is well-defined. To avoid ascertainment bias, patients selfcompleted QOL questionnaires independently from the clinical examination for postthrombotic syndrome. Finally, we systematically collected data on patient characteristics that could independently affect QOL, such as comorbidity and BMI, so that these factors could be adequately controlled for in our analyses.

The main limitations of our study relate to the fact that, by design, decisions regarding the duration of warfarin anticoagulation and the use of elastic compression stockings were left up to the treating physicians. Hence, we were not able to evaluate whether these factors may have independently influenced QOL.

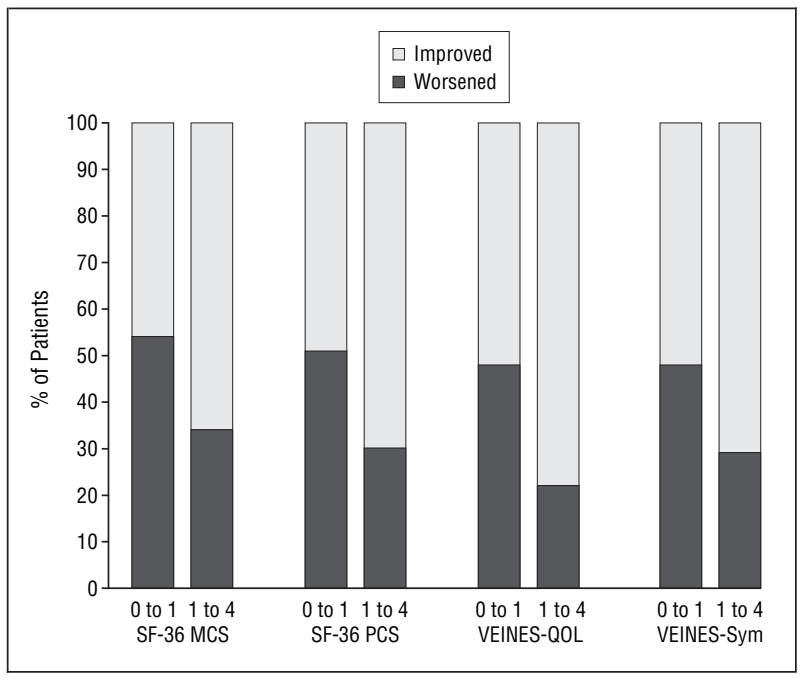

Figure 2. Proportions of patients during each interval who improved or worsened, by quality-of-life (QOL) measure. For the intervals, 0 to 1 indicates from baseline to 1 month; and 1 to 4 , from 1 to 4 months. Abbreviations are explained in the legend to Figure 1.

Table 3. SF-36 PCS and MCS Scores in Study Patients Compared With Other Populations*

\begin{tabular}{lll}
\hline Population & PCS Score & MCS Score \\
\hline $\begin{array}{l}\text { Study subjects } \\
\quad \text { Baseline }\end{array}$ & $38.7(12.7)$ & $46.4(11.8)$ \\
1 mo & $37.9(10.8)$ & $45.8(12.0)$ \\
$\quad 4$ mo & $43.0(11.6)$ & $50.4(10.5)$ \\
Canadian norms ${ }^{12} \dagger$ & $49.3(8.9)$ & $50.7(8.7)$ \\
US patients & & \\
$\quad$ With arthritis (mean age, 56.0 y) & $43.2(11.6)$ & $48.8(11.1)$ \\
$\quad$ With chronic lung disease & $42.3(14.1)$ & $44.5(12.3)$ \\
$\quad$ (mean age, 48.9 y) & $36.4(12.4)$ & $48.0(12.4)$ \\
$\quad$ With angina (mean age, 62.6 y) & &
\end{tabular}

Abbreviations: See Table 2

*Data are given as mean (SD).

†Standardized to the sex and age decile distribution of the Venous Thrombosis Outcomes Study population.

Few studies have reported on QOL after DVT. Most of these studies ${ }^{15-20}$ examined small numbers of selected patients, were cross-sectional or retrospective, lacked a baseline examination, or did not include a disease-specific QOL measure. Only 2 studies have examined longitudinal change in QOL in DVT patients, both with the aim of comparing effects of different DVT treatments. O'Brien and colleagues ${ }^{21}$ administered the SF-36 generic QOL measure at baseline and at 7 days after DVT diagnosis, and found that the physical functioning, role physical, and role emotional domains worsened to a similar degree, but social functioning worsened less, in patients treated at home vs in the hospital. During a randomized DVT treatment trial that compared low-molecular-weight heparin administered at home with intravenous heparin administered in the hospital, Koopman and colleagues ${ }^{22}$ evaluated change in QOL over 24 weeks after diagnosis using the Short Form-20 generic measure with the addition of a few questions on venous symptoms. As in our study, they found that QOL tended to improve over time, but proportions 
and characteristics of patients whose QOL remained poor during follow-up were not reported and a validated diseasespecific QOL measure was not used.

In conclusion, we found that on average, QOL improves during the 4 months following DVT, but remains poorer than that of population norms. Furthermore, about one third of patients have a decline in QOL during this period. Worsening of the postthrombotic syndrome score was independently associated with a decline in physical health status (SF-36 PCS score) and venous disease-specific QOL. Although widely considered to be an acute condition, our findings indicate that DVT has an adverse impact on QOL that persists beyond the short-term phase and provide evidence to support the evaluation of QOL in conjunction with clinical end points in trials comparing treatments for DVT. In addition, our study highlights the need for further research on pathophysiological mechanisms and clinical predictors of the postthrombotic syndrome.

Accepted for Publication: November 24, 2004. Correspondence: Susan R. Kahn, MSc, MD, FRCPC, Centre for Clinical Epidemiology and Community Studies, Sir Mortimer B. Davis Jewish General Hospital, 3755 Cote Ste Catherine, Room A-127, Montreal, Quebec, Canada H3T 1E2 (susan.kahn@mcgill.ca).

Funding/Support: The Venous Thrombosis Outcomes Study was supported by the Fonds de la Recherche en Santé du Québec, Montreal; and by an unrestricted grantin-aid from Sanofi Synthelabo, Paris, France. Drs Kahn and Shrier are recipients of Clinical Investigator Awards and Dr Johri is a recipient of an Investigator Award from the Fonds de la Recherche en Santé du Québec.

Role of the Sponsor: The study sponsors had no role in the design and conduct of the study; the collection, management, analysis, interpretation, and reporting of study data; the writing of the manuscript; or the decision to submit the manuscript for publication.

Previous Presentation: This study was presented in part at the American Society of Hematology 45th Annual Meeting; December 8, 2003; San Diego, Calif.

Acknowledgment: We thank the Venous Thrombosis Outcomes Study personnel at the clinical study sites for their dedication to this project.

\section{REFERENCES}

1. Lamping D. Measuring health-related quality of life in venous disease: practical and scientific considerations. Angiology. 1997;48:51-57.

2. Kahn SR, Solymoss S, Lamping DL, Abenhaim L. Long-term outcomes after deep vein thrombosis: post-phlebitic syndrome and quality of life. J Gen Intern Med. 2000;15:425-429

3. Patrick DL, Deyo RA. Generic and disease-specific measures in assessing health status and quality of life. Med Care. 1989;27:S217-S232.

4. van Korlaar I, Vossen C, Rosendaal F, Cameron L, Bovill E, Kaptein A. Quality of life in venous disease. Thromb Haemost. 2003;90:27-35.

5. Kearon C, Ginsberg JS, Hirsh J. The role of venous ultrasonography in the diagnosis of suspected deep venous thrombosis and pulmonary embolism. Ann Intern Med. 1998;129:1044-1049.

6. Lensing AW, Buller HR, Prandoni P, et al. Contrast venography, the gold standard for the diagnosis of deep-vein thrombosis: improvement in observer agreement. Thromb Haemost. 1992;67:8-12.

7. Hyers TM, Agnelli G, Hull RD, et al. Antithrombotic therapy for venous thromboembolic disease. Chest. 2001;119:176S-193S.

8. Villalta S, Bagatella P, Piccioli A, Lensing A, Prins M, Prandoni P. Assessment of validity and reproducibility of a clinical scale for the post-thrombotic syndrome [abstract]. Haemostasis. 1994;24:158a

9. Ware JE, Kosinski M, Keller S. SF-36 Physical and Mental Summary Measures: A User's Manual. Boston, Mass: Health Institute, New England Medical Center; 1994.

10. Lamping DL, Schroter S, Kurz X, Kahn SR, Abenhaim L. Evaluating outcomes in chronic venous disorders of the leg: development of a scientifically rigorous, patientreported measure of symptoms and quality of life. J Vasc Surg. 2003;37:410419.

11. Ware JE, Snow KK, Kosinski MA, Gandek B. SF-36 Health Survey: Manual and Interpretation Guide. Boston, Mass: Health Institute, New England Medical Center; 1993.

12. Hopman WM, Towheed T, Anastassiades T, et al; Canadian Multicentre Osteoporosis Study Research Group. Canadian normative data for the SF-36 health survey. CMAJ. 2000;163:265-271.

13. Kahn SR, Ginsberg JS. Relationship between deep venous thrombosis and the postthrombotic syndrome. Arch Intern Med. 2004;164:17-26.

14. Wiebe S, Guyatt G, Weaver B, Matijevic S, Sidwell C. Comparative responsiveness of generic and specific quality-of-life instruments. J Clin Epidemiol. 2003; 56:52-60.

15. Beyth RJ, Cohen AM, Landefeld CS. Long-term outcomes of deep-vein thrombosis. Arch Intern Med. 1995;155:1031-1037.

16. O'Donnell TF, Browse NL, Burnaud KG, Thomas ML. The socioeconomic effects of an iliofemoral venous thrombosis. J Surg Res. 1977;22:483-488.

17. Kahn SR, Hirsch A, Shrier I. Effect of post-thrombotic syndrome on healthrelated quality of life after deep venous thrombosis. Arch Intern Med. 2002; 162:1144-1148.

18. Schweizer J, Kirch W, Koch R, et al. Short- and long-term results after thrombolytic treatment of deep vein thrombosis. J Am Coll Cardiol. 2000;36:13361343.

19. Ziegler S, Schillinger M, Maca TH, Minar E. Post-thrombotic syndrome after primary event of deep venous thrombosis 10 to 20 years ago. Thromb Res. 2001; 101:23-33.

20. Comerota A, Throm R, Mathias S, Haughton S, Mewissen M. Catheter-directed thrombolysis for iliofemoral deep venous thrombosis improves health-related quality of life. J Vasc Surg. 2000;32:130-137.

21. O'Brien B, Levine M, Willan A, et al. Economic evaluation of outpatient treatment with low-molecular-weight heparin for proximal vein thrombosis. Arch Intern Med. 1999;159:2298-2304.

22. Koopman MM, Prandoni P, Piovella F, et al; Tasman Study Group. Treatment of venous thrombosis with intravenous unfractionated heparin administered in the hospital as compared with subcutaneous low-molecular-weight heparin administered at home. N Engl J Med. 1996;334:682-687. 